\title{
Chlamydia trachomatis Infection Challenge in Infants and Children
}

\section{Nanda Rachmad Putra Gofur ${ }^{1 *}$, Aisyah Rachmadani Putri Gofur², Soesilaningtyas ${ }^{3}$, Rizki Nur Rachman Putra Gofur ${ }^{4}$, Mega Kahdina ${ }^{4}$ and Hernalia Martadila Putri ${ }^{4}$}

${ }^{1}$ Department of Health, Faculty of Vocational Studies, Universitas Airlangga, Surabaya, Indonesia

${ }^{2}$ Faculty of Dental Medicine, Universitas Airlangga, Surabaya, Indonesia

${ }^{3}$ Department of Dental Nursing, Poltekkes Kemenkes, Surabaya, Indonesia

${ }^{4}$ Faculty Of Medicine, Universitas Airlangga, Surabaya, Indonesia

*Corresponding Author: Nanda Rachmad Putra Gofur, Department of Health, Faculty of Vocational Studies, Universitas Airlangga, Surabaya, Indonesia.

E-mail: nanda.rachmad.gofur@vokasi.unair.ac.id
Received: March 12, 2021

Published: June 15, 2021

(C) All rights are reserved by Nanda Rachmad

Putra Gofur., et al.

\begin{abstract}
Introduction: Babies born to mothers with active Chlamydia infection can have a 50 - 75\% risk of contracting it. This infection can affect the conjunctiva, nasopharynx, rectum and vagina of the baby. The most common clinical manifestation in neonates is inclusion conjunctivitis. Reported occurs in 15 - 37\% of babies born to mothers with cervical Chlamydia. Meanwhile, the part of the baby's body most often infected by Chlamydia is the nasopharynx. About 78\% of infants have positive nasopharyngeal cultures. Approximately half of the infants with inclusion conjunctivitis also become infected with the nasopharynx. C. trachomatis infection was also found in children with sexual abuse, with a rarer prevalence below $5 \%$. This infection is also often asymptomatic. The detection of infection in the rectum and vagina is usually found longer than infection of the conjunctivae and nasopharynx. Infections of the rectum and vagina are often asymptomatic. However, it can last for about 1 year.

Discussion: Several studies in the United States show that about 5 percent of neonates are infected with Chlamydia perinatally, but the prevalence of childhood antibodies before sexual activity is more than 20 percent. The incidence of childhood infections has not been well detected, but studies have found transmission from infected siblings (where the infection was acquired perinatally for more than 1 year) or from parents or other adults through sexual abuse. Infection in children can affect the upper respiratory tract of children, eyes, or middle ear infected with $C$. trachomatis may explain the difference in antibody levels in the neonatal period and adolescence, in which genital Chlamydia infection can be acquired by children as a result of sexual abuse. In comparison, an antibody cross-reaction against Chlamydia pneumoniae was performed which primarily infects the upper respiratory tract of children accounting for part or all of the seroprevalence of $C$. trachomatis.
\end{abstract}

Conclusion: Sexual abuse must be considered a cause of Chlamydial infection in infants and children. However, perinatally transmitted $C$. trachomatis infection of the nasopharynx, urogenital tract and rectum might persist for 2 - 3 years.

Keywords: Chlamydia Infection; Infant; Children; Manifestation 


\section{Introduction}

Chlamydia trachomatis is a pathogenic germ in humans and is one of four species with the genus Chlamydia. However, the species that infect humans are $C$. trachomatis, $C$. pneumonia and $C$. psittaci [1]. Each of these species contains many strains that have various serological and biological characteristics. The need for a better means of this specification is indispensable, especially for C. trachomatis. C. trachomatis species itself contains 4 biovariants which are different organisms. The Murine and Swine variants are not known whether they infect humans [1].

Epidemiological evidence strongly suggests that babies get Chlamydia infection from their mothers during labor. This is based on a number of prospective control studies of mother-child infection, where infection only occurs in infants born to infected mothers. No one has yet stated horizontal transmission from mother to child, from other family members to babies or from babies to other babies after delivery. Infection is rare during cesarean delivery. Infection that often occurs is in the case of premature rupture of the membranes so that contamination is possible [2].

Babies born to mothers with active Chlamydia infection can have a $50-75 \%$ risk of contracting it. This infection can affect the conjunctiva, nasopharynx, rectum and vagina of the baby. The most common clinical manifestation in neonates is inclusion conjunctivitis. Reported occurs in $15-37 \%$ of babies born to mothers with cervical Chlamydia. Meanwhile, the part of the baby's body most often infected by Chlamydia is the nasopharynx. About 78\% of infants have positive nasopharyngeal cultures. Approximately half of the infants with inclusion conjunctivitis also become infected with the nasopharynx [3].

C. trachomatis infection was also found in children with sexual abuse, with a rarer prevalence below $5 \%$. This infection is also often asymptomatic. The detection of infection in the rectum and vagina is usually found longer than infection of the conjunctivae and nasopharynx. Infections of the rectum and vagina are often asymptomatic. However, it can last for about 1 year [4].

\section{Discussion}

The study also found that about 33\% of infants with a positive isolation of nasopharyngeal infection developed pneumonia. The risk of developing pneumonia in babies born to mothers with Chla- mydia is reported to be $1-22 \%$. The risk of infection of the rectum and vagina in infants with Chlamydia mothers was reported to be $18.9 \%$ and $14.5 \%$. Several studies in the United States show that about 5 percent of neonates are infected with Chlamydia perinatally, but the prevalence of childhood antibodies before sexual activity is more than 20 percent. The incidence of childhood infections has not been well detected, but studies have found transmission from infected siblings (where the infection was acquired perinatally for more than 1 year) or from parents or other adults through sexual abuse [5].

Infection in children can affect the upper respiratory tract of children, eyes, or middle ear infected with $C$. trachomatis may explain the difference in antibody levels in the neonatal period and adolescence, in which genital Chlamydia infection can be acquired by children as a result of sexual abuse. In comparison, an antibody cross-reaction against Chlamydia pneumoniae was performed which primarily infects the upper respiratory tract of children accounting for part or all of the seroprevalence of $C$. trachomatis [6].

\section{Clinical manifestation of Chlamydia infection}

The main clinical manifestation of Chlamydia infection in neonates is neonatal conjunctivitis, of which $C$. trachomatis has been identified as the most frequent cause of the disease. Approximately $30-50 \%$ of babies born to mothers who have Chlamydia infection will develop conjunctivitis. This study has identified $C$. trachomatis in $10-40 \%$ of infants younger than 1 month old with conjunctivitis. The incubation period is 5 - 14 days after birth or earlier if there is premature rupture of membranes, approximately $50 \%$ of babies with conjunctivitis due to Chlamydia infection will also have nasopharyngeal infection [1].

The clinical manifestations vary widely, from mild conjunctivitis with dilute mucoid discharge to severe conjunctivitis with thick and purulent discharge, chemosis and pseudomembranous formation. The conjunctiva will be very fragile and bleed easily when swabbed. Edge of the eye erythema and often edema. Gram stain of the conjunctival swab shows predominance of PMN cells. Chlamydial conjunctivitis needs to be differentiated from gonococcal ophthalmic infection in some infants, especially in mothers who did not attend prenatal visits, received gonorrhea infection during pregnancy, and engaged in drug abuse. Neonatal conjunctivitis by Chlamydia has been suggested to induce long-term defects because 
it can lead to the formation of neovascular corneas and scars $[4,7]$.

The nasopharynx is the site of most Chlamydia infection at perinatal age. Nearly $70 \%$ of infected infants have positive cultures at this location, whereas nasopharyngeal infection itself is asymptomatic and can last for 3 years or more. Chlamydia pneumonia alone occurs in only $30 \%$ of infants infected with the nasopharynx. Infection of the nasopharynx is a secondary infection and conjunctivitis or is a direct infection that occurs in the nasopharynx. There are still differences of opinion. During labor, the possible source of infection comes from aspiration of infected cervical secretions. Results from prospective studies of mother-child infections have shown that pneumonia can occur in infants with Chlamydia conjunctivitis and it is believed the mechanism is the same. In infants with pneumonia, the clinical manifestations of pneumonia are very typical, including [8,9]:

- In children usually appear at the age of 4 - 8 weeks, some cases were reported to appear earlier, namely at week 2 but no cases appear more than 4 months of age.

- $\quad$ These babies usually have a history of coughing and access without any febrile.

- On physical examination found tachypnea and crackles on auscultation; wheezing is rare.

- On radiological images found only hyperinflammation that is not typical.

- Laboratory tests showed that the number of peripheral blood eosinophils increased ( $>300$ cells $/ \mathrm{cm}^{3}$ ) and increased serum immunoglobulin.

- In culture can be found the growth of microorganisms cultured from nasopharyngeal secretions.

Pneumonia in children caused by $C$. trachomatis is self-limited in nature. Most babies can be treated outpatient, but there are also some cases that need to be hospitalized because of obstruction and help with breathing. The diagnosis of $C$. trachomatis can be confirmed by serological examination. A 1:32 increase in IgM antibody titer is a strong sign of Chlamydia infection. In addition, C. trachomatis can be isolated from the nasopharynx. These infections are associated with decreased pulmonary function and airway obstruction, and infants with Chlamydia infection have a higher incidence of chronic respiratory distress than infants with non-Chlamydia infections [10].

\section{Laboratory examination}

Laboratory tests for Chlamydia have progressed rapidly over time. The ideal diagnostic test is one that has a sensitivity greater than $90 \%$ and a specificity greater than $99 \%$. The ideal test that should be used is nucleic acid amplification (AAN). However, the use of this test should be adjusted to the capacity of the available health facilities. For screening programs, a technique that uses a non-invasive specimen is usually used [11].

\section{Gram stain}

This technique is a faster laboratory test to evaluate urethritis and determine the presence or absence of gonococcal infection. Considered positive for NGU if there are more than 4 leukocytes with 1000 times magnification, but unable to see BE and BR [11].

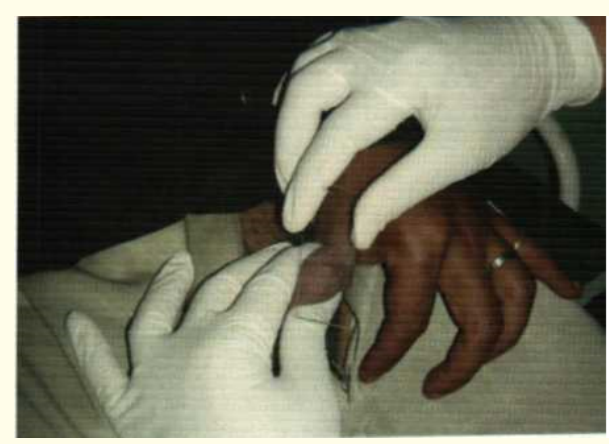

Figure 1: Collection of specimens for gram stain and wet [12].

\section{Urine sediment}

Urethritis diagnosis criteria are enforced if there is a urethral body and there are 20 or more PMN leukocytes in two or more of the five fields of view with 400x magnification from the sediment examination of $10-15 \mathrm{ml}$ of the first collected urine released before 4 hours or more [12].

\section{Leukocyte esterase test}

The approach to screening men with asymptomatic infection for the presence of polymorphonuclear leukocytes uses the urine leukocyte esterase (LE) test. The men were then tested specifically for Chlamydia infection or were treated empirically. Most investigators who use culture to determine Chlamydia infection, find the sensitivity of the LE test to predict Chlamydia urethritis is 41 to 85 percent among asymptomatic men, with a specificity of $75-95 \%$. 
Some authors conclude that the LE test is less sensitive as a means of screening [12].

Further studies are needed in populations universally screened with DNA amplification assays to clearly define the optimal role of LE in reducing costs. However, in circumstances where there are cost difficulties in implementing tests such as LCR and PCR, the use of an inexpensive LE test is likely to be cost effective, especially when combined with a simple assessment of risk in order to detect a higher probability of developing disease before testing.

\section{Cell culture}

Cell culture is the gold standard test for detecting C. trachomatis for several years or confirming the presence of this species. Sensitivity 40 - 85\% in genital specimens (cervix, urethra). The advantage is high specificity, both for medicolegal diagnosis purposes. The disadvantages of using cell culture [13]:

There are special requirements both in culture and in specimen transport techniques so that cell cultures can be cultured properly;

This technique is suitable for small amounts of invasive samples and requires specimen transport so it is impractical, difficult to perform and requires high costs.

\section{Antigen detection test}

The antigen detection test uses Chlamydia lipopolysaccharide (LPS) or major outer membrane protein (MOMP), as a means of detecting Chlamydia elementary bodies in genital specimens. The most widely used antigen detection tests are the direct fluorescent antibody assay (DFA) and enzyme immunoassays (EIA) assays [14].

\section{Direct fluorescent immunoassay (DFA)}

DFA has a sensitivity of $50-90 \%$ depending on skill and the number of elementary bodies in the specimen. The advantage of this technique is that it is suitable for both invasive and non-invasive specimens (e.g. urine). While the disadvantage is that this test is not suitable for large numbers of specimens and takes time [15].

\section{Enzyme immunoassays (EIA)}

This technique has a sensitivity of 20 - 85\% depending on the type of test. The advantage is that it can be used to examine large numbers of specimens, is fast and inexpensive. While the disadvantage is that it has a high sensitivity, only if a positive result is confirmed by other tests, it is only suitable for invasive specimens (cervix, urethra) [12].

\section{Nucleic acid hybridization}

This test has a sensitivity of $70-85 \%$, while the advantage is that it is fast and can be used automatically. Can be used for large samples. Tests can be performed to check the diagnosis of gonococcal infection at the same time. The disadvantage is that it can only be used for invasive specimens (cervix, urethra) $[12,15]$.

\section{Nucleic acid amplification (AAN)}

The AAN technique itself has a sensitivity of 70 - 95\%. This test has the advantage of high specificity (97 - 99\%), which can be used to examine large numbers of specimens. Its use can be on invasive specimens (cervix, urethra) or noninvasive (urine and vulvovaginal), it can be examined to diagnose gonococcal infection simultaneously. The disadvantage of this test is that it is expensive, it needs caution in use to avoid contamination in the laboratory. Chlamydia inhibitors, especially in urine specimens, can make this test difficult [13].

Currently there is an automated method for detecting DNA or RNA amplified C. trachomatis. The two most widely used methods are ligase chain reaction (LCR) and polymerase chain reaction (PCR). The other method is transcription-mediated amplification (TMA), propagates Chlamydial ribosomal RNA, and appears to have a similar action to LCR and PCR. The target of LCR and PCR is the nucleotide portion of the $C$. trachomatis plasmid, which is present in multiple copies of each elementary body. The TMA target is the ribosomal RNA portion. The lowest limit that can be detected by this test is 1 to 10 elementary bodies (compared to 10,000 elementary bodies for EIA) [14].

In order to determine the function of the new amplification tests, a standard other than cell culture is used, this requires reevaluation of specimens that are negative on culture and positive on amplification tests. The first confirmation used DFA on cytospin from culture transport media. If the results are negative with DFA, repeat the PCR or LCR again using the test against another target nucleotide part, called the chromosome MOMP gene sequence [15].

There is an alternative approach using 3 available amplification methods to confirm one amplification method using another similar method. Using the developed standard, the application of LCR to 
the urine of the first portion (10 - $14 \mathrm{ml}$ from the first stream) has a sensitivity of about 69 to $96 \%$ in detecting Chlamydial urethritis in women. The sensitivity of the LCR shown in endocervical specimens ranged from 81 - 100 [12,13].

Compared with cell culture results, LCR generally detects between 15 - 40\% infected patients, with an increase in prevalence of $4-5 \%$. One study indicated that the LCR shows that endocervical specimens are constantly more sensitive than culture, and that the LCR displays sufficient sensitivity to first-collected urine, meaning it is a noninvasive test that can be used to diagnose Chlamydia's urethral and cervical infection in women. Regarding the effect of inhibitors on endocervical specimens and urine on LCR and PCR, further research is needed [14].

Chlamydia is not significant for diagnosing acute urogenital infections. This is due to the high prevalence rate and the rare increase or decrease in IgG at the onset of infection, and patients are often in periods of absence of IgM. So that the serology test does not have much meaning. Usually, this serological test is widely used for the diagnosis of LGV and pneumonia in neonates [12].

Diagnosis and management of $C$. trachomatis infection in children

The diagnosis of Chlamydia ophthalmia was made based on Giemsa's stain of conjunctival scrap and found to be basophilic with intracytoplasmic inclusions. The correlation between cytology and Chlamydia culture in Chlamydia ophthalmia ranges from $39 \%$ to $90 \%$. This method has been replaced by culture and antigen detection tests, such as DFA, EIA and NAA. This test has a sensitivity of $>90 \%$ and a specificity of $>95 \%$ in conjunctival specimens compared with culture [16].

However, for nasopharyngeal specimens it is not very good. Although the sensitivity to detect $C$. trachomatis in nasopharyngeal specimens of infants with pneumonia is $>90 \%$, the sensitivity in conjunctivitis infants ranges from 38 to $91.7 \%$, DNA Probe, Pace II (Gen Probe) is more commercial, but noncultural tests for diagnosis Chlamydia infection has been used widely in several countries [17].

The goal of treatment is to prevent transmission to sexual partners. Therapy on sexual partners is also carried out to prevent reinfection. Therapy for children and infant is still carried out by preventing transmission to sexual harassment. Therapy Indications [18]:

- $\quad$ Established oculogenital C. trachomatis infection.

- C. trachomatis infection in sexual partners.

- If laboratory tests for C. trachomatis are not available, the patient has been confirmed as having Neisseria gonorrhoeae infection.

- If laboratory tests for $C$. trachomatis are not available, the patient shows clinical signs of Chlamydia infection.

The regimen for the treatment of non-specific genital infections caused by Chlamydia is to use [18]:

- $\quad$ Doxycycline 2 x $100 \mathrm{mg}$ per day orally, for 7 days OR.

- Azithromycin $1 \mathrm{~g}$ orally as a single dose OR.

- $\quad$ Tetracyclines 4 x $500 \mathrm{mg}$ per day orally, for 7 days OR.

- $\quad$ Erythromycin 4 x 500 mg per day orally, for 7 days.

The treatment is in accordance with the guidelines issued by the CDC-MMWR in 2015 regarding the management of Chlamydia infection, which is as stated below [8].

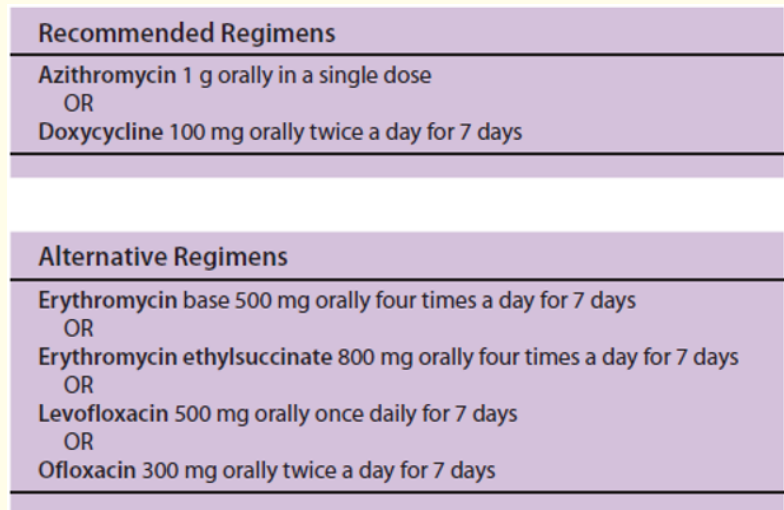

Figure 2: Guidelines for the therapy of Chlamydia infection according to the CDC - MMWR [18].

A test-of-cure culture (repeat testing after completion of therapy) to detect therapeutic failure ensures treatment effectiveness. 
Therefore, this culture with should be obtained at a follow-up visit approximately 2 weeks after treatment is completed [18].

\section{Conclusion}

Sexual abuse must be considered a cause of Chlamydial infection in infants and children. However, perinatally transmitted $C$. trachomatis infection of the nasopharynx, urogenital tract and rectum might persist for 2 - 3 years.

\section{Bibliography}

1. Lavett DK., et al. "Will the SAFE strategy be sufficient to eliminate trachoma by 2020 ? puzzlements and possible solutions". The Scientific World Journal (2013): 648106.

2. Bell TA., et al. "Risk of perinatal transmission of Chlamydia trachomatis by mode of delivery". Journal of Infection 29.2 (1994): 165-169.

3. Darville T. "Chlamydia trachomatis infections in neonates and young children". Seminars in Pediatric Infectious Diseases 16.4 (2005): 235-244.

4. American Academy of Pediatrics. Section 3: summaries of infectious diseases. chlamydial infections. In: Pickering LK, ed. Red Book: 2012 Report of the Committee on Infectious Diseases. 29th ed. Elk Grove Village, IL: American Academy of Pediatrics (2012).

5. Centers for Disease Control and Prevention. "Chlamydia Fact Sheet" (2013).

6. Centers for Disease Control and Prevention. Sexually Transmitted Disease Surveillance 2011. Atlanta, GA: US Dept of Health and Human Services (2012).

7. Davis CH., et al. "Protein disulfide isomerase, a component of the estrogen receptor complex, is associated with Chlamydia trachomatis serovar $E$ attached to human endometrial epithelial cells". Infection and Immunity 70 (2002): 3413-3418.

8. Sobel JD. "Vulvvaginal Candidiasis". In: Sexually Transmitted Disease. Holmes KK, Sparling PF, Mardh PA, Lemon SM, Stamm WE, Piot P, Wasserheit JN, eds. $3^{\text {rd }}$ edition. New York: The Mc Graw Hill Co, Inc (1999): 629-639.

9. Rosenman MB., et al. "Oral erythromycin prophylaxis vs watchful waiting in caring for newborns exposed to Chlamydia trachomatis". Archives of Pediatrics and Adolescent Medicine 157 (2003): 565-571.
10. Morbidity and Mortality Weekly Report (MMWR), Centers for Disease Control and Prevention. Sexually Transmitted Diseases Treatment Guidelines (2015).

11. Jacobson DL., et al. "Relationship of hormonal contraception and cervical ectopy as measured by computerized planimetry to chlamydial infection in adolescents". Sexually Transmitted Diseases 27 (2000): 313-319.

12. Gaydos CA., et al. "Chlamydia trachomatis infections in female military recruits". The New England Journal of Medicine 339 (1998): 739-744.

13. Thompson C., et al. "A family cluster of Chlamydia trachomatis infection". British Medical Journal 322 (2001): 1473-1474.

14. U.S. Preventive Services Task Force. Screening for chlamydial infection: U.S. Preventive Services Task Force recommendation statement". Annals of Internal Medicine 147.2 (2007): 128-134.

15. Laboratory Diagnostic Testing for Chlamydia trachomatis and Neisseria gonorrhoeae (2009).

16. Burstein GR., et al. "Expedited partner therapy for adolescents diagnosed with chlamydia or gonorrhea: a position paper of the Society for Adolescent Medicine". Journal of Adolescent Health 45.3 (2009): 303-330.

17. Gaydos CA., et al. "Sustained high prevalence of Chlamydia trachomatis infections in female army recruits". Sexually Transmitted Diseases 30 (2003): 539-544.

18. Niccolai LM., et al. "Pregnant adolescents at risk: sexual behaviors and sexually transmitted disease prevalence". American Journal of Obstetrics and Gynecology 188 (2003): 63-70.

\section{Volume 4 Issue 7 July 2021 (C) All rights are reserved by Nanda Rachmad Putra Gofur., et al.}

